\title{
EDITORIAL
}

\section{A mixed RECORD}

\author{
The recent publication of a large clinical trial indicating that the diabetes drug rosiglitazone \\ (Avandia; GlaxoSmithKline) does not increase the risk of cardiovascular morbidity and \\ mortality seems unlikely to end the controversy over its safety. Overall, the story highlights \\ the importance of risk communication in protecting public health.
}

In May 2007, a meta-analysis of clinical trials of rosiglitazone ${ }^{1}$ suggesting that it raised the risk of heart attacks created a media furore, with many stories emphasizing the reported $43 \%$ increase in relative risk. The impact was considerable: new prescriptions of the drug plummeted, the case featured prominently in arguments by FDA critics that the agency in general was 'broken', and it had a key role in catalysing the recent introduction of tougher FDA standards for the approval of new diabetes drugs.

Soon after the original meta-analysis - which involved pooling a group of trials that were not originally intended to explore cardiovascular outcomes - was published, interim data from a long-term trial known as RECORD, set up specifically to investigate the potential cardiovascular risks of rosiglitazone, were also reported ${ }^{2}$. The interim analysis found no evidence of an increase in death from cardiovascular causes linked to rosiglitazone, and the data were not sufficient to determine whether the drug was associated with an increased risk of heart attacks ${ }^{2}$.

Now, two years later, the final results of RECORD have been published ${ }^{3}$. The study involved 4,447 patients with type 2 diabetes receiving standard metformin or sulphonylurea monotherapy, who were randomly assigned to either addition of rosiglitazone $(n=2,220)$ or to a combination of metformin and sulphonylurea $(n=2,227)^{3}$. The primary end point was cardiovascular hospitalization or cardiovascular death, with a hazard ratio non-inferiority margin of 1.20 (20\% excess risk $)^{3}$.

During a mean 5.5-year follow-up, 321 patients in the rosiglitazone group and 323 patients in the active control group experienced the primary outcome ${ }^{3}$. Although the trial had less statistical power than initially planned owing to a lower event rate than anticipated, the hazard ratio for the primary outcome was 0.99 with a $95 \%$ confidence interval of $0.85-1.16$, excluding the predefined $20 \%$ excess risk and thereby satisfying the non-inferiority criterion $^{3}$. The previously known increased risk of heart failure associated with rosiglitazone was also confirmed ${ }^{3}$. In summary, the authors concluded: "Although our evidence is insufficient to rule out a small increased risk of myocardial infarction when compared with other glucoselowering agents, rosiglitazone does not increase overall cardiovascular morbidity or mortality."3
Such a conclusion might be considered surprising given the impact of the meta-analysis, and in this respect it is interesting to consider the limitations of the metaanalysis. For example, many of the trials analysed were small and short-term, and so had few adverse cardiovascular events or deaths. So, "the confidence intervals for the odds ratios for myocardial infarction and death from cardiovascular causes are wide, resulting in considerable uncertainty about the magnitude of the observed hazard." The odds ratio for myocardial infarction in the rosiglitazone group was 1.43 , with a $95 \%$ confidence interval of 1.03-1.98 (REF. 1).

Nevertheless, it was the ' $43 \%$ increased relative risk' that grabbed the headlines, and the effect of this on rosiglitazone use by patients with diabetes seems clearer. For example, a retrospective study of $\sim 13,000$ patients in the US Veterans Affairs Health System presented at the recent American Diabetes Association meeting (abstract 517-P) indicated that around half discontinued rosiglitazone therapy during a period in 2007 spanning the publicization of the potential safety risks of the drug. These patients experienced a significant increase in their glycated haemoglobin levels, indicating reduced glycaemic control.

Some commentators have criticized the design and validity of the conclusions of RECORD, and it seems unlikely that the issue of the cardiovascular risk of rosiglitazone will be resolved before an outcomes study against another drug in the same class, pioglitazone (Actos; Takeda), is completed in 2015. However, it is concerning that a meta-analysis reporting a risk with considerable uncertainty and important limitations has had such an impact on patients with diabetes and efforts to develop novel drugs for these patients. In this respect, it is encouraging that the new FDA leadership has recently emphasized both its commitment to balance the potential good of a medical product or policy against potential harm, and the key role of the agency in ensuring effective risk communication in its mission to protect public health ${ }^{4}$.

\footnotetext{
. Nissen, S. E. \& Wolski, M. P. H. N. Engl. J. Med. 356, 2457-2471 (2007).

Home, P. D. et al. N. Engl. J. Med. 357, 28-38 (2007)

3. Home, P. D. et al. Lancet 5 Jun 2009 (doi:10.1016/S01406736(09)60953-3).

4. Hamburg, M. A. \& Sharfstein, J. M. N. Engl. J. Med. 360, 2493-2495 (2009).
} 